\title{
Effect of a designed nursing protocol on nurse's knowledge and practice regarding Intravenous Therapy
}

\author{
Magda Abdo Thabet Ahmed, Zienab A. Mohammad, Manal E. Ez El-Deen, and Samia Youssef Sayed \\ Assistant lecturer of Medical-Surgical Nursing, Faculty of Nursing, Assiut University, Egypt \\ Professor of Medical-Surgical Nursing, Faculty of Nursing, Assiut University, Egypt \\ lecturer of Internal Medicine, Faculty of Medicine, Assiut University, Egypt \\ lecturer of Medical-Surgical Nursing, Faculty of Nursing, Assiut University, Egypt.
}

\begin{abstract}
:
Background: Peripheral venous cannulation is the commonest method used for intravenous therapy. There are numerous well recognized indications such as intravenous fluids, limited parenteral nutrition, blood and blood products, drug administration (continuous or intermittent). The aim of the present study is 3-folds: the first is to assess nurse's knowledge and practice regarding intravenous therapy, the second is to design nursing protocol for nurses about intravenous therapy and the third is to evaluate the effect of implementing the designed nursing protocol on nurse's knowledge and practice regarding intravenous therapy. Hypotheses: Three hypotheses were formulated: (1) the post mean knowledge scores of nurses who will be exposed to designed nursing protocol will be higher than their pre mean knowledge scores. (2) The post mean practice score of nurses who will be exposed to a designed nursing protocol will be higher than their pre mean practice scores. (3) A positive relationship will exist between knowledge and practice score obtained by nurses receiving the designed nursing protocol. Research design: Aquasi-experimental research design was utilized in this study on a convenient sample of 55 nurses. The study will be conducted in general medical departments (B and C) and blood diseases unite of Assiut university hospital. Tools: utilize for data collection were a) Questionnaire sheet. b) Observation checklist sheet. c) Construction of designed nursing protocol. Results: The first and the second hypotheses were supported as a good improvement in the mean knowledge \& practice scores were found after the implementing of designed nursing protocol. The third hypothesis was supported as a negative relationship was found between nurse's knowledge and practice scores immediately after application of the designed nursing protocol. Conclusion: implementing the designed nursing protocol on nurse's knowledge and practice regarding intravenous therapy shows a significant improvement in nurses' knowledge and practice. Recommendation: Continued nursing education and in-service training programs should be well organized within Assiut University Hospital.
\end{abstract}

\section{Key words: Intravenous therapy,knowledge,practice,nurses.}

\section{Introduction:}

Intravenous therapy (IV) is defined as the administration of fluids or drugs, or both, into the general circulation through a venipuncture which involves the administration of sterile preparations of drugs into venous blood. The aim of intravenous management is to be safe, effective delivery of treatment without discomfort or tissue damage and without compromising venous access, especially if long term therapy is proposed. (Jodim, et al., 2007)

Intravenous fluid and electrolyte therapy are commonly used to treat many different fluid and electrolyte imbalance. Many patients need maintenance IV fluid therapy only while they cannot take oral fluid (e.g., during and after surgery).Other patients need corrective or replacement therapy for losses that have already occurred. (Kee JL. et al., 2004)

The fact that $90 \%$ of hospitalized patients receive intravenous fluids and medication puts patients at risk for developing complications. Many of these intravenous complications can be avoid if nurse is a keen observer, knowledgeable and skillful.

Unfortunately, complicated cannulation may result in patients' deprivation from needed fluids or medications. Moreover, it will lead to increase in the hospital stay and subsequent increase in cost.

Therefore, expert nurses can play an active role in the prevention of cannulation complications throughout the precise cannulation steps. (Bergerson and Lopez, 2005)

Peripheral venous cannulation is the commonest method used for intravenous therapy. There are numerous well recognized indications such as intravenous fluids, limited parenteral nutrition, blood and blood products, drug administration (continuous or intermittent), prophylactic use before procedures and prophylactic use in unstable patients. Contraindications such as Inflammation or infection of the insertion site, forearm veins in patients with renal failure (may be needed for arteriovenous fistulae) and irritant drugs into small veins with low 
flow rates (that is, leg and foot veins). (Brunelle, 2003)

Intravenous catheters are used widely in modern medicine for therapy with fluid blood and blood products, medicine, oral nutritional support, and hemodynamic monitoring. In the United States, approximately 150 million intravenous catheters are used annually; most of them are reported to be peripheral catheters. (Erdem, 1998)

Veins on the non-dominant forearm are most suitable, especially if the cannula has to remain in position for any length of time. Veins on the dorsum of the hand are easiest to cannulate, but are more uncomfortable for the patient and more liable to block. Veins in the lower limb should be avoided where possible because of the increased incidence of thrombophlebitis and thrombosis. (Alexander, 2006)

Prepared IV solutions fall into three general categories: - isotonic hypotonic and hypertonic all IV fluids should be carefully given. The nurse must understand the rationale for IV fluid administration and the type of IV solution ordered because the names of IV solutions are often abbreviated or shortened. (Watson, 2010)

Intravenous therapy is so widely used that it is easy to take its safety for granted. Several potential complications of intravenous therapy can be very serious, however complications include local and systematic. Local complications including hematoma, thrombosis, phlebitis, Thrombophlebitis, infiltration, extravasation, local infection, and venous spasm. Systemic Complications including septicemia, fluid overload \& pulmonary edema, air embolism, speed shock, and catheter embolism. (Saloojee, et al., 2001)

Phlebitis is the most common complication of IV infusion therapy, and studies demonstrate that 20-80 percent of patients receiving peripheral IV therapy develop phlebitis. Phlebitis can be further classified into mechanical, chemical and infective phlebitis, depending on the cause of the problem. (Panadero, et al., 2010)

Treatment for phlebitis is usually heat and analgesia; however, the use of transdermal antiinflammatory gel is reported to be beneficial. The gel works as a vasodilator, counteracting vasoconstriction caused by the phlebitis. Antiinflammatory agents can be beneficial in reducing inflammation at the cannula site. (Philpot and Griffiths 2003) (Cokmez et al 2003)

Nurses have important responsibilities in intravenous catheter application. They administer intravenous fluid recommended by doctors, and they also perform subsequent treatment and observation. Nurses undertake additional important responsibilities such as starting, observing, and stopping the liquid flow, and the diagnosing and preventing complications caused by intravenous catheters. In general, these responsibilities comprise intravenous fluid treatment complications, symptoms and diagnosis of these complications, and applications that need to be used as soon as a complication develops. (Taxis and Barber 2003)

\section{The aims of the study:}

1. To assess nurse's knowledge and practice regarding intravenous therapy

2. To design nursing protocol for nurses about intravenous therapy

3. To evaluate the effect of implementing the designed nursing protocol on nurse's knowledge and practice regarding intravenous therapy.

Hypothesis:

To fulfill the aim of the study the following research hypothesis will be formulated:-

1. The post mean knowledge scores of nurses who will be exposed to design nursing protocol will be higher than their pre mean knowledge scores.

2 . The post mean practice scores of nurses who will be exposed to designed nursing protocol will be higher than their pre mean practice scores.

3. A positive relationship will exist between knowledge and practice score obtained by nurses receiving the designed nursing protocol

\section{Significance of the study:}

From the researcher's clinical experience it has been observed that intravenous therapy is one of the most common invasive procedures performed in hospitals and a major cause of morbidity. More than 25 million patients have peripheral intravenous catheters placed each year in United States hospitals (Saloojee et al., 2001). Infusion therapy is believed to account for one third of all nosocomial bacteremia .Intravenous complications increase morbidity, inpatient stay, hospital cost and mortality of patients, which makes the health care providers do great efforts to decrease the incidence of these complications .Nurses are the largest group of workers and they have close contact with patient, They are uniquely placed to incorporate preventive and promote strategies in day -to-day care they provide. Therefore, they should have knowledge, good attitude and skillful in dealing with these complications. So this study will be carried out to assess their knowledge and practice regarding intravenous therapy.

\section{Subjects and Methods}

Research design: quasi-experimental research design was utilized in this study.

Setting: The study will be conducted in the general medical departments (B and C) and blood diseases unit at Assiut University Hospital. 
Sample: All available nurses working in general medical departments B \&C (45) and nurses working in blood diseases unit (10) at Assiut university hospital and who were participate in the study.

\section{Tools:}

Tool- I: Pre/post nurse's knowledge questionnaire sheet: consisted of for parts:

Part (1): Socio- demographic data for nurses.

Part (2): Nurses' knowledge about intravenous therapy.

Part (3): Nurses' knowledge about intravenous injection.

Part (4): Nurses' knowledge about intravenous solutions.

Tool II: Nurse's practice observation checklist sheet: It consists of the following seven main procedures:-

1. Selection of veins

2. Intravenous injection

3. Venipuncture using needle or catheter

4. Preparing an I.V. solution

5. Adding medication to an IV solution

6. Starting intravenous infusion

7. Discontinuing an intravenous infusion

Tool III: Construction of a designed nursing protocol: It was developed according to the needed nurse's knowledge and practice that can help nurses in provision of a safe care for patient. Including main topics:

knowledge: anatomy of veins, definition of intravenous therapy, Indications of intravenous therapy(injection, fluid), advantages and disadvantages of intravenous therapy, types of intravenous fluids, complications of intravenous therapy(definition, causes, signs and symptoms ,preventive measures and nursing intervention for each complication ) , nursing care of intravenous therapy(before ,during ,after.)

Practices: Selection of veins, intravenous injection procedure (preparation and administration), I.V Catheter (insertion and maintaining I.V catheter), I.V solution (preparation, administration of I.V solution and adding medication to I.V solution).

Scoring system:

Each right answer was given one score. The total scores of knowledge were 72 and the total scores of practices were 112,each item was observed, categorized and scored into either done $=1$,or not done $=0$.

$<50 \%$ unsatisfactory level from $50 \%: 70 \%$ satisfactory level

$>70 \%$ good level

Ethical approval:

Each nurse was informed with the purpose of the study. The investigator emphasized that the participation is voluntary and confidentially and anonymity of subjects will be assured through coding of all data. Verbal consent was obtained from each nurse prior to his/her contribution in the present study. Confidentiality of any obtained information was secured.

Methods:

The study was carried out on 3 phases:-

Phase I: Preparatory phase

The study tools were designed after extensive literature review.

The content and validity was done by expertise opinion in medical and nursing field.

A pilot study was conducted on $10 \%$ of sample in a selected setting to evaluate the applicability and clarity of developed tools.

Phase II: Implementation phase

An official permission will be obtained from the head of medical departments to conduct the study.

Nurse's and patient's agreement for voluntary participation was obtained and the purpose and nature of the study will be explained.

Each nurse involved in the study was assessed for his or her knowledge using the written knowledge test (tool 1).

Each nurse was observed directly while performing Procedure (tool II).

Application of designed nursing protocol was performed by researcher then by nurses (tool III).

Phase III: Evaluation phase

Post test was performed to evaluate nurse's knowledge and practice regarding I.V therapy using tool (I \& II) immediately post test and after one month post test.

Data analysis:

Data were analyses using SPSS. The following tests for significance were used, Frequency, Percentage, Means and Standard Deviation. Chi square (with yets correction). Correlation coefficient, ANOVA and t-test for comparison of means. A probability level of 0.05 was adopted as a level of significance for testing the research hypothesis.

$\mathrm{P}>0.05$ non significant

$\mathrm{P}<0.05$ significant

$\mathrm{P}<0.005$ moderate Significant

$\mathrm{P}<0.001$ highly significant 


\section{Results:}

Table (1): Frequency distribution of socio demographic characteristics of studied nurses:

\begin{tabular}{|c|c|c|}
\hline Variable & No. $(n=55)$ & $\%$ \\
\hline \multicolumn{3}{|l|}{ Sex: } \\
\hline - Male & 17 & 30.9 \\
\hline - Female & 38 & 69.1 \\
\hline \multicolumn{3}{|l|}{ Age: } \\
\hline - $<30$ years & 18 & 32.7 \\
\hline - $\quad \geq 30$ years & 37 & 67.3 \\
\hline - $\quad$ Mean \pm SD (Range) & \multicolumn{2}{|c|}{$33.87 \pm 6.40(18-45)$} \\
\hline \multicolumn{3}{|l|}{ Marital status: } \\
\hline - $\quad$ Single & 7 & 12.7 \\
\hline - Married & 48 & 87.3 \\
\hline \multicolumn{3}{|l|}{ Education: } \\
\hline - Nursing diploma & 51 & 92.7 \\
\hline - Nursing technical Institute & 4 & 7.3 \\
\hline \multicolumn{3}{|l|}{ Years of experience: } \\
\hline - $<10$ years & 11 & 20.0 \\
\hline - $10-<20$ years & 25 & 45.5 \\
\hline - $\quad \geq 20$ years & 19 & 34.5 \\
\hline - $\quad$ Mean \pm SD (Range) & \multicolumn{2}{|c|}{$15.11 \pm 5.90(2-25)$} \\
\hline \multicolumn{3}{|l|}{ Previous training courses } \\
\hline - Infection control & 19 & 34.5 \\
\hline - Intravenous therapy & 9 & 16.4 \\
\hline - $\quad$ None & 27 & 49.1 \\
\hline \multicolumn{3}{|l|}{ Numbers of traning courses } \\
\hline - One & 18 & 64.3 \\
\hline - Two or more & 10 & 34.7 \\
\hline \multicolumn{3}{|l|}{ Benefits from training courses } \\
\hline - Good & 19 & 67.9 \\
\hline - Very good & 9 & 32.1 \\
\hline
\end{tabular}


Table (2): Total and subtotal mean knowledge score levels obtained by nurses pre, immediately and one month after application of a designed nursing protocol. $(\mathrm{n}=55)$

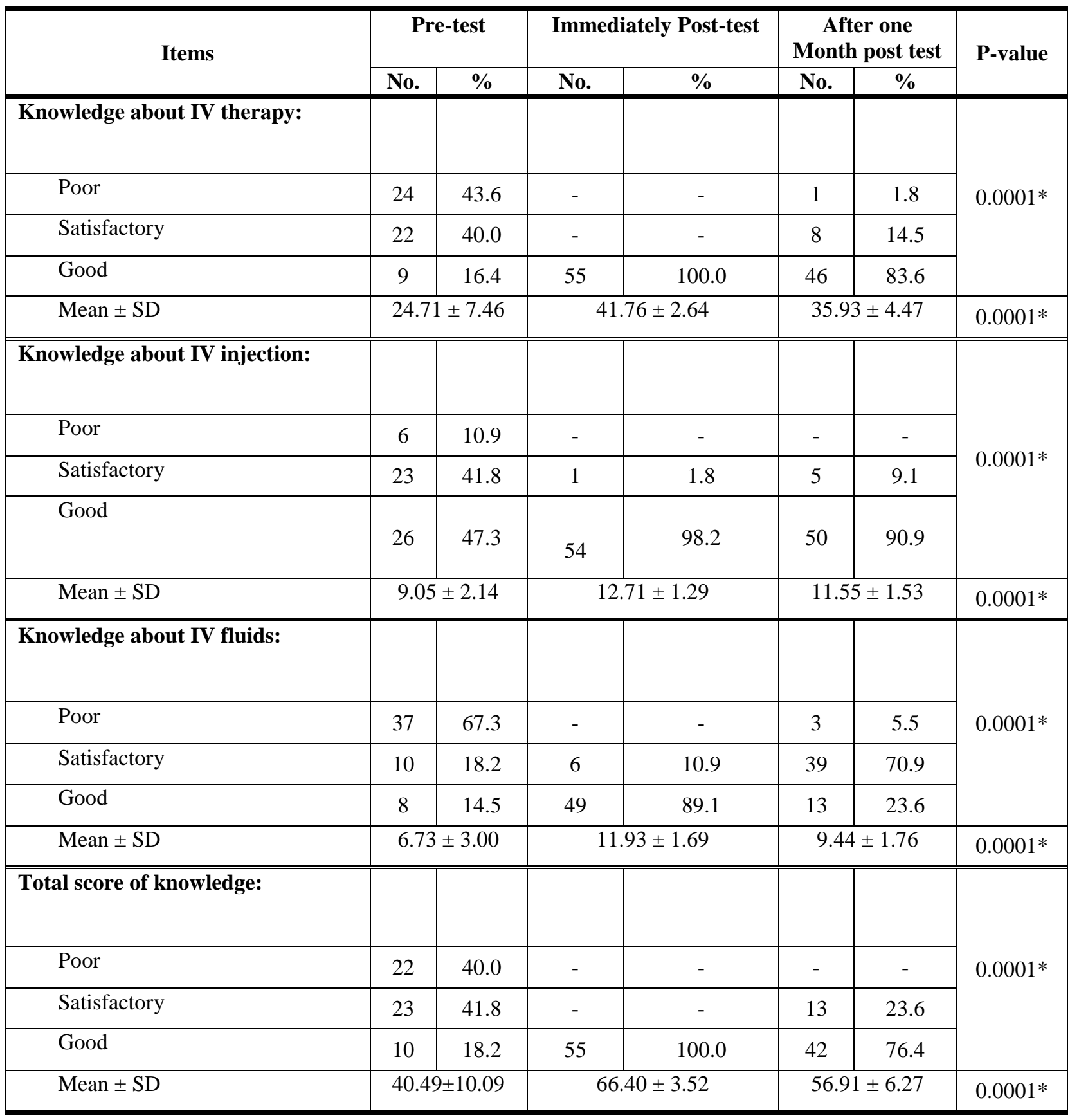


Table (3): Total mean practice scores obtained by nurses pre-, immediately and one month after application of a designed nursing protocol $(n=55)$

\begin{tabular}{|c|c|c|c|c|c|c|c|}
\hline \multirow{2}{*}{ Practices } & \multicolumn{2}{|c|}{ Pre-test } & \multicolumn{2}{|c|}{ Immediately Post-test } & \multicolumn{2}{|c|}{$\begin{array}{c}\text { After one } \\
\text { month post test }\end{array}$} & \multirow{2}{*}{ P-value } \\
\hline & No. & $\%$ & No. & $\%$ & No. & $\%$ & \\
\hline Poor & 41 & 74.5 & - & - & - & - & \multirow{3}{*}{$0.0001 *$} \\
\hline Satisfactory & 14 & 25.5 & 10 & 18.2 & 33 & 60.0 & \\
\hline Good & - & - & 45 & 81.8 & 22 & 40.0 & \\
\hline Mean \pm SD & \multicolumn{2}{|c|}{$53.82 \pm 7.28$} & \multicolumn{2}{|c|}{$81.65 \pm 3.49$} & \multicolumn{2}{|c|}{$76.42 \pm 5.95$} & $0.0001 *$ \\
\hline
\end{tabular}

Table (4): Correlation between nurse's knowledge and practices in pre, post, and after one month

\begin{tabular}{|l|c|c|}
\hline & r-value & P-value \\
\hline Pre-test & -0.077 & 0.574 \\
\hline Immediately Post-test & 0.042 & 0.763 \\
\hline After one month post test & -0.138 & 0.315 \\
\hline
\end{tabular}

Table (1) shows that: The majority of nurses $(67.3 \%)$ their age were more than 30 years. $(87.3 \%)$ of them were married $(45.5 \%)$ of them had $10-<20$ years of experience. $(69.1 \%)$ of them were female. (92.7\%) of them had diploma of nursing. As regard to training in specialty field, it was found that $(65.5 \%)$ of nurses not attended training course of infection control.

Table (2) shows that: The majority of nurses had good level of knowledge post implementing a designed nursing protocol (100.0) with $(\mathrm{P}<0.0001)$.

Table (3) shows that: The baseline mean scores for total practice pre implementing of a designed nursing protocol (53.82 \pm 7.28), However, a good improvement in the mean practice scores post implementing of a designed nursing protocol (81.65 \pm 3.49 ) and the mean practice scores after one month of implementing of designed nursing protocol (76.42 \pm 5.95).A significant relation between nurse's practice in relation to mean practice score with $\mathrm{P}<0.0001$ Table (4) shows that: There was no positive correlation between nurses' knowledge and practice in pre-, immediately post-test and one month posttest.

\section{Discussion}

Based on the results of the present study, the majority of the nurses their ages were more than 30 years, married, female, and have diploma of nursing, the majority of them had in-service training courses related to infection control but they had no in-service training courses related to intravenous therapy and their experience range from 10 to less than 20 years.

Marquis and Huston (2009): stated that education and training are two components of staff development that occur after an employees' indoctrination (which refers to planned, guided adjustment of employee to the organization and work environment). The staffs' knowledge level and capabilities are a major factor in determining the number of staff required to carry out unit goals. The better trained and more competent the staff, the fewer the staff required, which in turn saves the organization money and rise reproductively.

As regards nurse's knowledge about intravenous therapy the results revealed that there is statistically significant difference in majority of items on pre and immediate post-test. This result in line with Ahmed (2011) who documented that data collected before the designed nursing protocol implementation (pre-test) showed unsatisfactory level of knowledge about renal failure, hemodialysis and care of patients undergoing hemodialysis, which reflected the lack in their scientific preparation. The implementation of the designed protocol showed an improvement in the nurses' level of knowledge regarding the care offered to hemodialysis patients. The findings indicated that a good improvement in the mean knowledge scores after the application of the designed nursing protocol.

By time after one month post-test, the percentage was slightly reduced as the majority of nurses were having satisfactory and good levels in all items of knowledge. This indicates that the improvement in knowledge was partially lost one month after implementation of teaching protocol. This result might be explained by the fact that, knowledge retention is usually affected by time. 
Broomfield (1996) conducted a research with the intention of testing the retention of certain nursing skills and knowledge of registered nurses. There has been an initial improvement after performing the training program, but there has been a significant decrease in retention of knowledge 10 weeks later $(\mathrm{P}<0.0001)$. The findings of his research reflected similar results to previous research works, suggesting that retention of skills and knowledge quickly deteriorates with time if not used or updated regularly. He recommended refreshing courses on regular basis.

In this regards, Mehany (1999) and AbdAlla (2000), found a direct relation between memory loss and length of time that lapses after a certain educational event. Also they reported that nurses who had poor levels of knowledge and/or skills before the exposure to a training program underwent a significant improvement after the implementation of the program.

The results in the present study revealed that there was a great improvement in the practice score levels obtained by nurses after the application of the designed nursing protocol. This has been concluded by the presence of significant difference between results of pre-test and post-tests. This finding indicated that skills can be easily improved, especially if linked with their relevant scientific base of knowledge.

Youssef, (2007); in the same line with the current study findings conducted a study at the reconstructive microsurgical \& trumatology care units in Assiut University Hospital. Entitled "Microvascular free tissue transfer surgeries, Impact of a designed teaching protocol on nurse's knowledge, practices and patient's outcome" which revealed that an improvement in nurse's practice after the attendance at continuing nursing education sessions. Research findings indicated that continued nursing education programs increase both knowledge and practice and can also improve attitudes.

In this respect $\boldsymbol{A b d - A l l a , ~ ( 2 0 0 0 )}$ documented that the inservice training program has a beneficial effect in improving the nurses' knowledge and skills. As well as, Ookalkar, (2009) who recommended that educational programs should be organized according to the needs of nurses with continuous evaluation and adopting proper checklists for work monitoring to enhance patient and staff awareness; led to reduced process errors, mitigating overall risks, eventually resulting in effective patient care.

The results in the present study showed that there was no significant difference between nurses' knowledge and their practice observed during the pre-test. This may be attributed to insufficient courses related to intravenous therapy included in their undergraduate curriculum of nursing education and also there is no available Arabic source for updating and continuing their education.

Also the finding of (WHO, 2002) which found that in order to maintain and improve the quality of patient care, continuous data collection, documentation and analyzing patient information before, during and after intravenous therapy is essential.

Finally, it can be concluded that, the designed nursing protocol for nurses working with intravenous therapy has achieved, its objectives by improving nurses' knowledge and practice regarding intravenous therapy. This further was supported by (Porth, 2000); who stated that professional nurses have a large role to play in the minimization and prevention of intravenous complications and should be clinically well versed in all aspects of the condition, current strategies to address risk minimization and prevention management and advocates for patient safety.

\section{Conclusion:}

Based on the results of the present study, it can be concluded that:

- Nurse's knowledge and practice regarding intravenous therapy in general medical departments (B and C) and blood diseases unit at Assiut university hospital are inadequate. Nurses are potentially capable of improving their knowledge and practice after exposure to a designed nursing protocol.

- Implementing the designed nursing protocol on nurse's knowledge and practice regarding intravenous therapy shows a significant improvement in nurse's knowledge and practice.

- There is no standard of care provided pre, during, and post intravenous therapy.

Recommendations:

Based on the results of the present study the following can be recommended:

For nurses:

1. Continued nursing education and inservice training programs in general medical departments (B and C) and blood diseases should be well organized within Assiut University Hospital and equipped with the necessary educational facilities and materials necessary to upgrade the knowledge and skills of practicing nurses, which will be reflected on better outcome and service for inpatients.

2. Nurses should add to their routine obligations the regular reading of up-to-date references (periodicals, textbooks, etc.). They should always be encouraged to attend scientific meetings and conferences to keep pace with the 
rapidly growing wealth of knowledge and practice necessary for proper nursing service.

3. Newly employed nurses' in general medical departments (B and C) and blood diseases unit are required to successfully complete a test of basic knowledge and skills before assuming independent responsibility for patient care.

For administration:

1. Adequate supplies and facilities should be available in the unit.

2. Periodic monitoring of nurses knowledge and practice to evaluate the level of nurses.

3. Orientation program for all newly nurses and in services training programme for experienced nurses about intravenous therapy.

4. Availability of manual procedures for nurses to be aware of handling any problems that may arise.

5. It is recommended to prepare a placement for hand washing in the unit as (sink, antimicrobial soap, paper towels for drying).

6. Following routine testing policies; hepatitis $\mathrm{B}$ and $\mathrm{C}$ and HIV antibodies for patients and staff.

For further research:

Replication of the study on a larger probability sample acquired from different geographical areas in Egypt to figure out the main aspects of this problem.

\section{References:}

1. Abd-Alla M (2000): Assuring quality care through a managerial inservice training program for head nurses working in Assiut University Hospital. DNS thesis of nursing service administration. Assiut University.

2. Ahmed G.H., (2011): Effect of designed nursing protocol on nurse's knowledge and practice regarding Hemodialysis patients, submitted for partial fulfillment of Master Degree in Medical and surgical Nursing Faculty of Nursing, Assiut University.

3. Alexander M. (2006): Infusion nursing standards of practice. Journal of Intravenous Nursing, 29 (IS), S1-S92.

4. Bergerson L, Lopez $V$ \& Molassiotis A., (2005): An Intervention study to evaluate nursing management of peripheral intravascular devices. Journal of Infusion Nursing; 24(2):11323..

5. Broomfield R., (1996): A quasi-experimental research to investigate the retention of basic cardiopulmonary resuscitation skills and knowledge by qualified nurses following a course in professional development. JADV NURS, 23(5): 1016-23.
6. Brunelle D., (2003): Impact of a dedicated infusion therapy team on the reduction of catheter related nosocomial infection. Journal of infection Nursing. 26(6), 362-366.

7. Cokmez A, Gur S, Genc H, Deniz S \& Tarcan E., (2003): Effect of transdermal glyceryl trinitrate and anti-inflammatory gel in infusion phlebitis. ANZ Journal of Surgery .pp. 73, 10, 794-796.

8. Erdem I., (1998): The evaluation of intravascular catheter infections. Infect $\mathrm{J} ; 13: 31$ 4.

9. Jodim \& Willard., (2007): Introduction to Medical Surgical Nursing, 6thed, Saunders Company, p.p277:283.

10. Kee J.L., Paulanka, B.J., \& Purnell L.D., (2004): Fluids and electrolytes with clinical applications ( $7^{\text {th }}$ ed.). Clifton Park, NY: Delmar Cengage Learning.

11. Marquis L. Bessie, Huston J. \& Carol (2009): Leader ship roles \& management functions in nursing, $6^{\text {th }}$ ed., Lippincott, Hong Kong, p. 371.

12. Mehany M (1999): Effect of basic emergency care program on emergency nurses performance. DNS thesis in medical surgical nursing, Assiut University.

13. Ookalkar, Anil G. Joshi, Dhananjay S. \& Ookalkar., (2009): "Quality improvement in hemodialysis process ", International Journal of Quality \& Reliability Management, Vol. 26 Iss: 8, pp.817-830.

14. Panadero A, Iohom G, Taj J, Mackay N \& Shorten G (2010): A dedicated intravenous cannula for postoperative use: effect on incidence and severity of phlebitis. Anesthesia. 57, 9, 921-925.

15. Philpot $P$ \& Griffiths V (2003): the peripherally inserted central catheter. Nursing Standard. 17, 44, 39-46.

16. Porth C., (2000): Pathophysiology: Concepts in altered health states. $5^{\text {th }}$ edition. New York. Lippincott Company.

17. Saloojee H. and Steenhoff, A., (2001): The health professional's role in preventing nosocomial infections .Postgraduate Medical Journal77 (903)16-19.

18. Taxis $K$ \& Barber N., (2003): Ethnographic study of incidence and severity of intravenous drug errors. British Medical Journal. 326, 7391, 684.

19. Watson and Osborn. (2010): Medical Surgical Nursing, Pearson education, p.p512-538.

20. WHO. (2002): Fifty -fifth world health assembly Quality of care: patient safety Report 
by the Secretariat Medication A55/ 13 Provisional agenda item 13.923.

21. Youssef S.S., (2007): Micro vascular free tissue transfer surgeries Impact of a designed teaching protocol on nurse's knowledge, practices and patient's outcome. Submitted for fulfillment of the requirements of Doctorate Degree in Adult Nursing, Faculty of Nursing, Assiut University. 